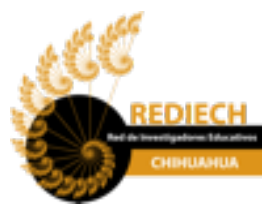

Red de Investigadores Educativos Chihuahua A.C. Chihuahua, México www.rediech.org

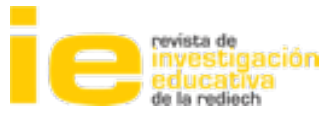

ISSN: $2007-4336$

ISSN-e: 2448-8550

http://www.rediech.org/ojs/2017/index.php/ie rie rediech/index

\author{
Francisco Alberto Pérez Piñón \\ Stefany Liddiard Cárdenas \\ Guillermo Hernández Orozco
}

2019

\title{
Fundamentos teórico-metodológicos en la investigación educativa en Chihuahua; análisis de un área del conocimiento
}

IE Revista de Investigación Educativa de la REDIECH, 10(18), pp. 143-160.

http://dx.doi.org/10.33010/ie rie rediech.v10i18.521

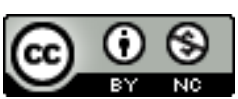

Esta obra está bajo licencia internacional

Creative Commons Reconocimiento-NoComercial 4.0.

CC BY-NC 4.0 


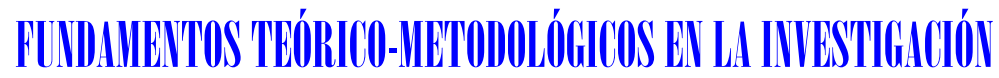

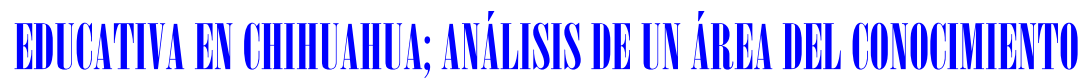

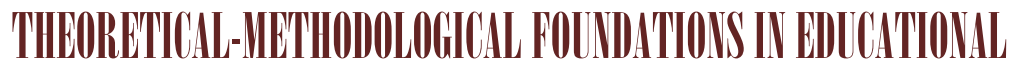

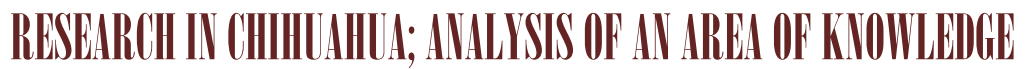

\author{
PÉREZ PIÑÓN Francisco Alberto \\ LIDDIARD CÁRDENAS Stefany \\ HERNÁNDEZ OROZCO Guillermo
}

Recepción: diciembre 28 de 2018 | Aprobado para publicación: marzo 30 de 2019

DOI: https://dx.doi.org/10.33010/ie_rie_rediech.v10il8.521

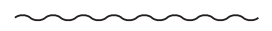

\section{Resumen}

El objetivo del presente artículo es mostrar el análisis de las tendencias teóricas y metodológicas investigativas del área denominada "Teoría, filosofía, historia e historiografía de la educación e investigación sobre la investigación educativa". Se argumenta con el análisis de las ponencias presentadas en dos congresos de investigación educativa organizados por la Red de Investigadores Educativos Chihuahua. Se consideran las instituciones participantes a través del perfil laboral de los autores, la

Francisco Alberto Pérez Piñón. Profesor-investigador de la Universidad Autónoma de Chihuahua, México. Entre sus publicaciones recientes están: "Apuntes para una historia posmodernista" en Espacio Abierto (2016) y "La Hacienda de Coyotillos, un acercamiento a los contenidos educativos" en Acercamientos a la historia de la educación. Diálogos, actores y fuentes en la construcción del conocimiento histórico (2017). Cuenta con reconocimientos Prodep y del Sistema Nacional de Investigadores. Es miembro de la Sociedad Mexicana de Historia de la Educación, del Consejo Mexicano de Investigación Educativa y de la Red de Investigadores Educativos Chihuahua. Correo electrónico: aperezp@uach.mx. ID: http://orcid.org/0000-0003-4316-6484.

Stefany Liddiard Cárdenas. Profesora de la Universidad de Ciencias del Comportamiento, Chihuahua, México. Entre sus publicaciones recientes están: "Herramientas de la sociología de la educación aplicadas en la comunidad mormona Le Barón en Chihuahua", en Sociológica (2017) y "Representaciones sociales: Colonia Le Barón, Chihuahua" en Acercamientos a la historia de la educación. Diálogos, actores y fuentes en la construcción del conocimiento histórico (2017). Es socia activa de la Red de Investigadores Educativos Chihuahua y de la Sociedad Mexicana de Historia de la Educación. Correo electrónico: stefanyliddiard@gmail.com. ID: https:/orcid.org/0000-0002-3234-4372.

Guillermo Hernández Orozco. Profesor-investigador de la Universidad Autónoma de Chihuahua, México. Entre sus publicaciones recientes están: "José Joaquín Calvo López, fundador del Instituto Científico y Literario, hoy Universidad Autónoma de Chihuahua" en IE Revista de Investigación Educativa de la REDIECH (2017) y Debates por la historia (cinco tomos). Cuenta con reconocimientos Prodep y del Sistema Nacional de Investigadores nivel I. Es miembro de la Sociedad Mexicana de Historia de la Educación y de la Red de Investigadores Educativos Chihuahua. Acreedor al Reconocimiento Eduardo Flores Kastanis a la Investigación Educativa en 2018. Correo electrónico: ghernand@uach. mx. ID: http://orcid.org/0000-0001-7287-8240. 
mecánica de trabajo desarrollada en los congresos y finalmente se realiza un acercamiento teórico centrado en los paradigmas de la investigación histórica, identificando los elementos presentes en las ponencias de historia e historiografía de la educación, ya sea desde el historicismo, la escuela francesa de los annales, la interdisciplinariedad o el paradigma crítico de la historia.

\title{
Palabras clave: METODOLOGÍA DE LA INVESTIGACIÓN, INVESTIGACIÓN EDUCATIVA, HISTORIA DE LA EDUCACIÓN.
}

\begin{abstract}
The objective of this article is to show the analysis of theoretical and methodological research trends in the area of theory, philosophy, history, the historiography of education, and research on educational research. The analysis was made through the papers presented in two conferences of educational research organized by the Network of Educational Researchers in Chihuahua. The participating institutions are considered through the work profile of the authors, the work mechanics developed in the congresses and finally a theoretical approach centered on the paradigms of historical research, identifying the elements present in the papers of history and historiography of the education, whether from historicism, French School Annales, interdisciplinarity or the critical paradigm of history.
\end{abstract}

Keywords: RESEARCH METHODOLOGY, EDUCATIONAL RESEARCH, HISTORY OF EDUCATION.

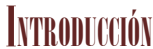

\section{Haciendo una}

síntesis de los antecedentes de estos congresos y la razones por las cuales se analizaron en esta área de investigación las tendencias metodológicas, se comienza por mencionar que las instituciones convocantes a los congresos que a continuación se narran fueron iniciativa de los miembros de la Red de Investigadores Educativos Chihuahua (Rediech), creada en el año 2010 por un grupo de entusiastas profesores-investigadores que buscaban una instancia que trascendiera la vida institucional, así como la forma de organizar las actividades que se daban al interior, ya que en ocasiones existía duplicidad de actividades. Como ejemplo de ello, y como un caso concreto, estaba la presentación de los avances de investigación a final de cursos semestrales en cada institución, por lo que se pensó que podría ser de mayor aprendizaje si se realizaban de manera interinstitucional; derivado de ello se comenzó de manera bianual la realización de un congreso, convertido en el 2018 en congreso internacional.

En esta ocasión solo se tomaron en cuenta dos congresos. Uno de ellos fue el 144 III Congreso Nacional de Investigación Educativa, llevado a cabo del 10 al 12 de 
noviembre del 2016 y denominado Aportes y debates para la construcción de una agenda educativa; ciertamente el título es concordante con la pretensión de estas actividades: impactar en las políticas públicas y que la investigación desarrollada sea considerada como parte de la política educativa estatal y nacional. Este congreso se catalogó como nacional por la afluencia de asistentes, ponentes, conferencistas y panelistas que en su mayoría provinieron de diversas partes de la República Mexicana. Las instalaciones de la Universidad Autónoma de Ciudad Juárez cobijaron a los participantes.

Si bien existió una distribución mayor de las diferentes áreas investigativas, aquí se analiza solamente el área que agrupa a la teoría, filosofía, historia e historiografía de la educación e investigación sobre la investigación educativa, aclarando que, con fines organizativos y debido a la poca cantidad de ponencias, en la programación de ambos congresos fueron unidas, pero debido a la naturaleza y especialización de cada una de ellas, en la práctica las mesas fueron separadas en diferente tiempo y espacio.

El IV congreso denominado Logros y desafios de la investigación educativa como herramienta para la transformación social tuvo carácter de internacional, debido a que en esta ocasión hubo presencia de invitados, conferencistas, panelistas y ponentes de distintos países. Se contó con personas de nacionalidad española, colombiana, guatemalteca, estadunidense y cubana, lo que fomentó una mayor diversidad de las ideas relacionadas con la investigación educativa. Este intercambio permitió el acercamiento a nuevos conocimientos y conocer distintos sustentos epistemológicos, teóricos y metodológicos, enriqueciendo la investigación educativa y su reconocimiento como actividad cotidiana, para que los actores educativos se conviertan en investigadores de su propia práctica docente (Morán, 1993). Las actividades de este evento se desarrollaron del 11 al 13 de octubre del 2018; su sede fueron las instalaciones de la Facultad de Filosofía y Letras y de la Facultad de Artes, ambos de la Universidad Autónoma de Chihuahua.

Ahora bien, en cuanto las ponencias presentadas en ambos congresos, mayoritariamente fueron colectivas, lo que quiere decir que se elaboraron por dos o tres autores; esto debido a las políticas educativas vigentes en educación superior -donde está el grueso de la investigación-, pues se les recomienda realizar trabajo colaborativo entre los profesores afines a sus líneas de investigación, o bien el trabajo conjunto entre miembros de cuerpos académicos, en donde pasan a ser, en palabras de Garay (2009, p. 18):

Producto de las políticas públicas impulsadas por el gobierno federal desde el sexenio pasado para todas las instituciones públicas de educación superior del país. Se trata de problematizar la manera en que las organizaciones educativas [...] atienden la complejidad de las formas de organización de la investigación que conviven en su interior.

Esto conduce a reflexionar que en el estado de Chihuahua y tardíamente, las instituciones obedecen estas políticas, generando mayor competencia entre los docentes, quienes deberán dedicarse a la investigación, y que una de las formas para producir colegiadamente es crear y/o pertenecer a un cuerpo académico, avanzando entre los grados que se establecen dentro de estos mediante su evaluación, que según el 
Programa de Mejoramiento del Profesorado (2018) pueden ser tres: el primer nivel es "en formación", el segundo "en consolidación" y el tercero es "consolidado". Es por ello que los investigadores acatan los términos de producción académica y alcanzar los niveles establecidos.

Otro aspecto adicional es la exigencia de obtener el perfil deseable por el Programa de Desarrollo Profesional. Se trata de un reconocimiento a docentes de tiempo completo que cubren una serie de requisitos y funciones en educación superior; 0 bien a cuerpos académicos en busca de fortalecimiento. Se establecen convocatorias anuales. Este programa emite convocatorias a los profesores de tiempo completo en las que deberán comprobar docencia, la generación y aplicación del conocimiento, realizar gestoría académica y contribuir siendo tutor. Estas políticas públicas y formas de destinar el presupuesto para la educación colocan a los profesores en una eterna competencia, por lo que es común escuchar lo que circula en corrillos en el medio magisterial acerca del número de ponencias, publicaciones, perfiles, cuerpos académicos, reconocimientos y diferentes funciones sustantivas en la investigación, como pertenecer al Sistema Nacional de Investigadores (SNI).

Es innegable que se está generando una cultura en las instituciones en las que para poder obtener apoyos, recursos y reconocimientos es necesario acatar las disposiciones oficiales o políticas educativas establecidas, pero no de manera informal, sino con un convencimiento de que es la manera de avanzar en este nivel educativo tanto a nivel individual con el profesorado, como a nivel institucional con la suma contabilizada de los perfiles (Prodep), los cuerpos académicos y el número de investigadores pertenecientes al SIN.

En Chihuahua, y dentro del nivel superior, un factor que ha influido en el cumplimiento de estas normas ha sido la Red de Investigadores Educativos Chihuahua, ya que permite a los profesores complementar el trabajo docente con la investigación, incluso contando con un espacio de publicación, donde se deja constancia por escrito y es posible la comunicación de productos académicos investigativos que han elaborado algunos miembros de la red. Ahondando en el tema de la revista, en el comienzo sirvió para difundir la producción de los investigadores locales, pero en estos años ha trascendido fronteras y se encuentra indexada a catálogos de gran impacto, reconocidos en el área de las ciencias sociales, nacionales y latinoamericanos, que permite una mayor visibilidad a nivel mundial y publicando en ella la producción de diferentes personajes desde diversas latitudes que desean difundir, todas sobre investigación educativa.

Como resumen de lo escrito anteriormente, a la fecha en las instituciones de educación superior, ya sean públicas, autónomas, institutos tecnológicos, escuelas normales y de educación básica, tienden a realizar el trabajo de forma colegiada, buscando mayor difusión. En esta carrera, que como política educativa ha sido asimilada como parte del desarrollo del profesorado y de la calidad de la educación, sigue sin ser nada sencillo para lograr los propósitos establecidos en dichas políticas; esto se logra entender desde dentro, al escuchar quejas sobre la gran carga de actividades asignadas a los profesores, siempre objeto de comentarios. 


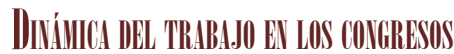

Como en la mayoría de los congresos en las ciencias sociales, las principales actividades a desarrollar han sido las conferencias magistrales. Para esto se selecciona previamente al conferencista, quien expone una serie de argumentos, y al final es posible interactuar con el público con una serie de preguntas y respuestas, también sancionado según el tiempo considerado para la actividad. También coexisten las presentaciones de libros, en la cual una o dos personas dan a conocer las temáticas del libro y se motiva para que realicen su lectura, dándole oportunidad al público asistente a que realice comentarios o preguntas. Otras más son los simposios 0 sesiones paneles, en donde un grupo de expertos expone temáticas definidas con anterioridad y al finalizar se presenta la oportunidad de preguntar o comentar por el público básicamente el mismo esquema en los tres. Si el congreso provee los espacios y recursos suficientes se muestran las exposiciones de libros, que tienen el fin de dar a conocer textos publicados, pudiendo ser en algunos casos de la autoría de los asistentes y ponentes al congreso. Finalmente, y motivo del presente análisis, son las presentaciones de ponencias, llamando la atención la dinámica realizada en estos dos congresos, ya que se han promovido algunos cambios innovadores.

Precisamente es en muchos de los coloquios o congresos en el área de las ciencias sociales, específicamente en educación, ya sean de corte nacional o internacional, lo habitual es presentar un informe por parte del o los ponentes, según sea el caso planificado por los autores y el tiempo límite para su exposición, utilizando generalmente algún apoyo visual, siendo al final de la ronda de ponencias cuando se dedica un tiempo para que los asistentes realicen preguntas y/o comentarios.

Generalmente, las ponencias se agrupan por afinidad temática y son de cuatro a cinco ponencias, contando entre 15 y 30 minutos a cada ponente para exponer su trabajo. Esta mecánica ha sido objeto de señalamientos, indicado que no es lo mejor, ya que el ponente está tan preocupado por su exposición o porque lo escuchen, que se pierde la atención hacia los otros participantes; 0 como ha ocurrido en ocasiones, ponentes solo hacen presencia cuando es su turno de presentar su trabajo y luego se van, algo así como un turismo académico. Este esquema de presentación se experimenta en los congresos tan reconocidos a nivel nacional como los que organiza el Consejo Mexicano de Investigación Educativa (COMIE) y el de la Sociedad Mexicana de Historia de la Educación (Somehide); se menciona esta experiencia por la magnitud y prestigio de ambas asociaciones.

Ante esta situación, dichos comentarios evaluativos se consideraron por los miembros de la Rediech, coincidiendo en que el esquema quedó agotado, y fue así que en una de las reuniones ordinarias de evaluación -que regularmente tienen lugar después de un congreso- se propuso romper con ello e innovar a través de formas distintas a las ya narradas. Fue así que desde el tercer congreso se planteó la distribución de mesas temáticas, buscando la afinidad de los trabajos presentados $\mathrm{y}$ evaluados para su presentación en el congreso, sin saturar el número de participantes, con el fin de generar procesos dialógicos que permitan la interacción entre quienes presentan sus productos. Para lograr esto, es necesario nombrar a un moderador, quien previamente a realizar su función lee la totalidad de los trabajos que 
se presentan y elabora preguntas direccionadas, logrando con ello la participación con respuestas ante cuestionamientos, pero pueden ser interpelados por los mismos ponentes o por el público asistente, quien puede pedir la ampliación de la temática, hacer preguntas o comentarios; además del tiempo para explicar su trabajo, pero sin el encuadramiento anterior.

De esta dinámica establecida en los últimos dos congresos se rescatan resultados favorecedores, percibiendo mayor motivación tanto para los ponentes como para el público en general, quienes ya no permanecen pasivos, sino que pasan a ser activos dentro del proceso dialógico en el que se exponen temas, inquietudes y consideraciones, todas en beneficio de la investigación educativa. En esta libertad impera la buena función del moderador, quien mantiene el ánimo participativo y evita monopolizar la palabra; el hecho que el moderador conozca previamente los temas permite imprimirle una dinámica que conduce al abundamiento de lo tratado en cada mesa de trabajo.

El cambio hacia este nuevo formato se presentó posterior a la discusión durante las reuniones colegiadas entre los miembros de la asociación civil Rediech. Dicha modificación fue aprobada como una innovación para poner nuevamente en movimiento las ideas investigativas, como algo más interesante que rebase el acartonamiento de describir pausadamente los descubrimientos o hallazgos; esta modalidad ha sido evaluada en ambos congresos, considerándose como una mejora. Se refirma que en esta dinámica la fuerza intelectual que le imprime el moderador es decisiva en el interés y abundamiento de la temática, tanto de los ponentes como de los asistentes a la mesa de trabajo.

Ahora bien, existen similitudes en cuanto a la organización de los congresos organizados por el COMIE y por la Rediech, debido a que ambas se enfocan en la investigación educativa. Por una parte, las áreas temáticas fueron un referente a seguir, clasificadas conforme a las siete propuestas por el Consejo Mexicano de Investigación Educativa (2015):

Teoría, filosofía, historia y educación e investigación sobre la investigación educativa; Diversidad, interculturalidad y sustentabilidad en la educación; Políticas y gestión en la educación; Currículum, conocimientos y prácticas educativas; Procesos de formación y actores de la educación; Tecnologías de la información y la comunicación en educación; Valores, convivencia, disciplina y violencia en la educación.

De estas se modificó únicamente el área "diversidad, interculturalidad y sustentabilidad en la educación", agregando el "género" dentro de la misma. Por otra parte, se utiliza bajo renta el sistema o software para el envío, asignación de árbitros, revisión, con lo que se agiliza el trabajo. Asimismo, con este sistema se obtiene la emisión automatizada de constancias a todos los participantes, independientemente de la función que hayan realizado durante el proceso.

De las áreas mencionadas anteriormente se explicita a continuación de manera sintética la nombrada teoría, filosofía, historia y educación e investigación sobre la investigación educativa, área objeto de análisis del presente artículo. Para que 148 las ponencias sean catalogadas en esta área deben referirse a las teorías, debates 
ontológicos y epistemológicos referenciados al campo de la educación, políticas educativas, formación ciudadana; investigaciones de historia e historiografía de la educación, todos ellos conferidos en un amplio espectro, pero dentro de lo teórico, filosófico, histórico o investigación de la investigación educativa.

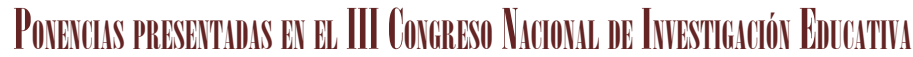

El análisis de ambos congresos se divide a su vez en tres apartados correspondientes. El primero de ellos es teoría y filosofía de la educación; en segundo lugar, historia e historiografía de la educación; y, finalmente, investigación de la investigación educativa.

En el primer apartado de teoría y filosofía de la educación se encuentran las ponencias: "Derechos humanos. Abstracción, homogeneización y dominación", de Juan Durán; "La formación del ciudadano desde la hermenéutica de lo ausente", por Norma Leticia Rodríguez; y "Lo imaginario y lo concreto en las formaciones discursivas sobre la figura del maestro mexicano", presentada por Leonardo Meza y Tomás Ávila. En este caso, todos los autores son profesores-investigadores de dos campus de la Universidad Pedagógica Nacional del Estado de Chihuahua.

Al desentrañar las ponencias se afirma que la concepción de una ausencia de lo humano está desapareciendo cada vez más en los espacios escolares, haciendo necesario visibilizar tanto las formas de dominación que ocurren en su interior, como su análisis. De ahí "la importancia de asumir una actitud hermenéutica que logre llevar a la visibilidad los factores que en el proceso de formación del ciudadano han sido invisibilidades por intereses y permanecen en la ausencia" (Rodríguez, 2016, p. 59). La intención, vista de manera general, es formar un ciudadano consciente de lo que ocurre a su alrededor, apoyándose en argumentos teóricos como los de Walter Benjamin, Manuel Reyes Mate y Ricardo Forster.

Con su ponencia, Juan Durán (2016) pone de manifiesto la necesidad de vivir los procesos áulicos de formación educativa, abandonar las prácticas pedagógicas del olvido por parte de los sujetos educativos y tener en cuenta el principio de no considerarlos como simples objetos, junto a la insuficiencia de experimentar pedagogías más focalizadas en los sujetos.

Asimismo, rescatar y poner en práctica mediante la discusión y análisis de las narrativas discursivas que se han elaborado del maestro mexicano, con la invisibilización de los actores educativos, quienes operan como si no formaran parte del proceso humanizado educativo, por ello se pretende trascender la hermenéutica de lo ausente por una hermenéutica de lo presente. No se descuida la influencia de Walter Benjamin con sus deseos y apreciaciones por pensar a la sociedad desde la marginalidad, desde lo periférico, en el cual están los principales males de la sociedad, dejando en el olvido a los desposeídos, tal como si no pensaran, otorgándoles lo peor en el caso de la educación.

Las aportaciones teóricas en este apartado son relevantes, citando particularmente a estos autores que dan sustento a los trabajos. En cuanto a la metodología de la investigación, está dividida en dos partes: una teórica, la predominante, y otro 
segmento empírico basado en entrevistas, resultado del trabajo de campo. Indudablemente estas ponencias correspondientes a la teorización, la filosofía y el campo de la educación se ocupan de las preocupaciones del siglo XXI, como la deshumanización de los procesos educativos, la pérdida del sujeto pensante por un depositario de saberes, la cosificación de sujetos a-históricos en donde el tiempo vivencial es olvidado por otro tiempo que es fabricado del futuro, ese que no se detiene ni olvida los males del pasado, como una forma de cerrar los horizontes temporales o simplemente de olvidarlos.

En las argumentaciones y posturas de la ponencia relacionada con la figura del maestro, Meza y Ávila (2016) mencionaron el enaltecimiento y el camino recorrido de las funciones que se le han asignado en el transcurso de la historia; hace recordar que, a inicios de la educación, en la época independiente se comenzó con la del apostolado mexicano, aquel profesor que debía ser un apóstol de la educación, capaz de sacrificarse por los demás, fundirse en las comunidades y sacarlos del ostracismo cultural. Durante el periodo cardenista, o de la educación socialista, con la función del activista político capaz de negociar aguas, bosques y tierras en bien de las clases desposeídas y teniendo como ideal construir una sociedad igualitaria. Después del socialismo la función cambia, y en la época del presidente Miguel Alemán se trató de recuperar la confianza en la sociedad mexicana, por lo que el profesor fue un factor de unidad nacional, un promotor del patriotismo y de los símbolos creados como iconos de mexicanidad.

Otra de las funciones que evoca el planteamiento de los discursos acerca del profesor es el de un trabajador más y reducido al trabajo áulico, donde lo que sucede fuera de las aulas no cuenta como labor magisterial; incluso cómo actualmente el profesor está propenso a cambios de actividad debido a las evaluaciones constantes del que son sujetos. En sí, dentro de los discursos de la llamada reforma educativa se coloca al profesor como el único responsable de la baja calidad de la educación, sin considerar otros aspectos estructurales. Los teóricos en que se sustenta la ponencia "Lo imaginario y lo concreto en las formaciones discursivas sobre la figura del maestro mexicano" son reconocidos en el campo de la historia: Carlo Ginzburg como padre de la microhistoria italiana, que permite visualizar espacios pequeños, y Fernando Braudel como uno de los representantes de la escuela francesa de los annales, referenciado para entender las historias de larga, media y corta duración.

Las tres ponencias proporcionan aportaciones al campo de la educación al estar recuperándolas mediante el análisis, para que no se pierdan en una simple memoria. En relación a los autores, profesores de la Universidad Pedagógica Nacional del Estado de Chihuahua, obliga a virar y concluir que la línea o líneas de formación estudiantil en esta institución serán como el discurso lo menciona: críticos, analíticos, reflexivos y propositivos.

En el segundo apartado de la historia e historiografía de la educación se presentaron siete ponencias. Cuatro de ellas provienen de docentes y estudiantes de la Facultad de Filosofía y Letras de la Universidad Autónoma de Chihuahua. Las ponencias "Al rescate de contenidos histórico-educativos: la Hacienda de Coyotillos" y "La escuela unitaria "Agustín de Iturbide' de Sueco, Chihuahua, 1945-1952" y "Memoria 150 e identidad. La recuperación de la historia educativa de Ranchería Juárez a través 
de sus protagonistas" fueron presentadas por los doctores Jesús Adolfo Trujillo, Guillermo Hernández y Francisco Alberto Pérez en diferente orden de autoría, pero presentes en las tres. También se ubicó la ponencia "La diáspora de los mormones fundamentalistas: Colonia Le Barón, Chihuahua, por Stefany Liddiard Cárdenas y dos de los doctores ya mencionados.

En este mismo apartado, pero adscritos al Centro de Investigación y Docencia, se presentaron con la ponencia "La educación física en la ciudad de Chihuahua 19001950" por Blanca Estela Chávez, Federico J. Mancera y Lilia Rey. Otra ponencia presentada por maestros del Centro Chihuahuense de Estudios de Posgrado fue "La instrucción pública en la nueva escuela de primeras letras en la ciudad de Chihuahua en 1869. Un intento de innovar", por José Guadalupe Ramos, Carmen Griselda Loya y Arturo Vázquez. Una más, titulada "Historia cultural de la educación especial en Chihuahua: transformación de su cultura escolar 1970-2015", la presentó Fernando Ponce, de la Universidad Pedagógica Nacional del Estado de Chihuahua.

En este apartado de historia de la educación ubican elementos teóricos y metodológicos de microhistorias descriptivas con enfoques culturales del estado de Chihuahua. Dichos trabajos realizados (Trujillo, Hernández y Pérez; Hernández, Trujillo y Pérez; Pérez, Hernández y Trujillo, 2016) resultaron de la consulta de fuentes documentales de archivos, pero considerando también las fuentes orales, como en el caso de la recuperación de la historia de Ranchería Juárez.

La mayoría parten de enfoques de la historia clásica o del tiempo pasado, y con menor frecuencia se observan trabajos interdisciplinarios. Por ejemplo, en una de ellas está presente un enfoque histórico y sociológico con la vida cotidiana desde la perspectiva de Agnes Heller, rescatando significados y cuestiones icónicas o representaciones sociales, manteniéndose en la línea de la historia cultural a la manera de Peter Burke, como lo trabajó Liddiard, Pérez y Hernández (2016), lo que enriquece y eleva lo empírico a un plano teórico fundamentado como temática de la vida real.

De manera sintética, es posible rescatar dos posturas, mismas fuertemente debatidas hasta el día hoy: por una parte, la recuperación o reconstrucción de los acontecimientos pasados con fines educativos y de identidad de los chihuahuenses; $y$, por otro lado, la que problematiza la temática con juicios subjetivos; esta se acerca más a la historia de la cuarta generación de la escuela francesa de los annales que, incluso, podrían ubicarse dentro de una postura posmodernista de la historia, con la cual se pretende rescatar al sujeto como artífice y creador de sus propias circunstancias, como se explicita en una de las ponencias: dar voz a los que no tienen voz.

Abundando en el análisis, las exposiciones de Chávez, Mancera y Rey, Ramos, Loya y Vázquez, y Ponce, aportan no solo a la historicidad, sino también a la diversidad de información útil para conformar y reafirmar tanto la herencia cultural como la identidad de los chihuahuenses. Algo que define a estas ponencias es la pasión por la historia, además de las contribuciones al rescate de lo historiográfico, teniendo la firme idea de que por medio de la historia de la educación se puede tener una mejor sociedad, en la que se reconozcan las vetas y se pueda construir un entorno basado en el respeto y paz, contando con los satisfactores necesarios para la reproducción de la vida material y espiritual. 
En este análisis se identifican paradigmas historiográficos con una marcada tendencia del historicismo rankeano, aquel que se enfoca en la traducción o paleografía de documentos o huellas del pasado, reconstruyendo los acontecimientos tal como se dijeron; sin embargo, se divisa el uso del planteamiento a partir del surgimiento de la escuela francesa de los annales, con la cual se introdujo un elemento ineludible hoy en día: la interpretación basada en las evidencias encontradas para dar un plusvalor a los estudios en este campo; esto al apreciar en algunos trabajos la interdisciplinariedad con la sociología, la psicología y la antropología, disciplinas que permiten el abundamiento en la obtención de información que se busca, lo que los sitúa en la posmodernidad, en la cual el cerco epistémico unidisciplinar queda caduco al abrir las fronteras disciplinares y los préstamos metodológicos para llegar a la esencia de lo investigado.

En el tercer y último apartado asignado a la investigación sobre la investigación educativa se presentaron cuatro ponencias. Una de ellas fue "EEducación moral para la autonomía? Fundamentos y contradicciones", de Betzabeth Reyes, de la Universidad Nacional Autónoma de México. Otra más titulada "Deserción universitaria en ingeniería en sistemas computacionales: el caso de la Universidad Politécnica Metropolitana de Puebla", por Elvia Bautista, de la Benemérita Universidad Autónoma de Puebla. Una tercera llamada "Educación vacía: un reto en la zona del Bajío, de Juan Manuel Torres, proveniente de la Universidad Nacional Autónoma de México. Finalmente, la ponencia "Evaluación de los índices de reprobación de la universidad usando intervalos de confianza", por Héctor Francisco Ponce, de la Universidad Autónoma de Ciudad Juárez.

En estas cuatro ponencias sobre la investigación sobre la investigación educativa contienen temáticas abordadas por un solo ponente, a diferencia de las otras áreas. Investigar a profundidad lo relacionado con la ética desde el enfoque kantiano, como la actuación del hacer el bien sin mirar a quién, o de actuar bajo el imperativo categórico de la idea de lo justo, pero sin el descuido de la realidad, es que en ocasiones los teóricos de la ética no son confiables. Sin duda, estar en el campo educativo significa actuar correctamente; al menos esa practicidad en el contexto es algo que no se debe soslayar, ni tampoco la autonomía del sujeto tratado, que desde el mencionarlo como sujeto se debe a la sujeción de las normas y reglas cívicas.

Como se ha enunciado, además de las limitantes sociales, políticas, económicas y culturales, existen procesos de selección para ingreso o tener acceso a la educación formal, justificándose en que identifican las características necesarias para el ingreso a estudiar alguna profesión y tener menos deserción o reprobación. También es cierto que existe un alto índice de reprobación, como lo expresado en la ponencia de las ingenierías en sistemas computacionales, derivando en la entrega de apoyos y estímulos a los estudiantes, precisamente para que no abandonen los estudios. Sin embargo, el problema de la deserción escolar es parte del mismo sistema de redistribución o de reproducción de las profesiones y ocupaciones en un sistema económico, político y social capitalista.

La teoría de la reproducción social, con la cual Pierre Bourdieu analiza la realidad, elude a la necesidad de reproducir la misma cantidad de obreros campesinos 152 y profesionistas para que la sociedad funcione, o al menos desde este enfoque es- 
tructuralista y/o funcionalista las deserciones escolares serían necesarias, pero el ser profesor o estar ubicado en el campo educativo obliga a la búsqueda de opciones para retener al mayor número de estudiantes en las distintas carreras o profesiones.

Las temáticas versadas se encuentran en dos planos. Por una parte, dos de las ponencias abordan la reprobación y las deserciones en el nivel superior. Bautista y Ponce, además de exhibir el desiderátum de investigarlos, también enfrentan estas problemáticas mediante la propuesta de estrategias para que se desdibujen 0 al menos se reduzcan. Por otra parte, las otras dos ponencias están perfiladas a mostrar una educación vacía, esa que se publica en los diarios, que desde la postura de Moscovici incide en las representaciones sociales; 0 bien, en el imaginario respecto a la educación, por lo que es urgente recuperar la esencia de la formación, concebir al docente como un ser humano encargado de formar a otros y teniendo como herramienta tanto a la educación como la recuperación de la autonomía de los sujetos desde una educación para la moral (Reyes, 2017 y Torres, 2016).

En cuanto a los autores, se desempeñan como profesores de prestigiadas universidades del país, resultando interesante el origen y la preocupación con la que abordan las temáticas. Se asegura que de tomar en cuenta lo que se aborda en estos documentos, la educación mejoraría; sin embargo, se ha corroborado que aquellos que toman las decisiones educativas no voltean a ver lo investigado y expuesto en congresos como estos, descuidando la sinergia que puede realizarse entre actores educativos y aquellos responsables en las políticas públicas. Sin duda estas cuatro ponencias contribuyen al acrecentamiento del conocimiento y expresan propuestas enfiladas a la calidad de la educación, una calidad tan debatida y necesaria actualmente.

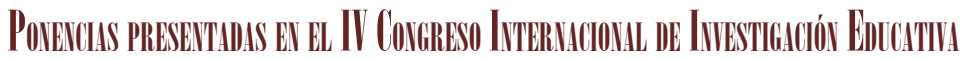

Realizado en Chihuahua capital en 2018, se presenta el análisis de la misma área, organizado como el apartado anterior, resultando que en el primer apartado de teoría y filosofía de la educación se presentaron dos ponencias. Una de ellas, titulada "La filosofía como método de enseñanza y aprendizaje en la educación primaria", presentada por Gabriela Alejandra Contreras, Laura Cristina Baca y Adela Cardona, de la Escuela Normal del Estado de Chihuahua; la segunda llamada "Ingesting power, ingerir poder" de Eva Marxen y Adam J. Greteman, procedentes de la School of the Art Institute of Chicago, de Norteamérica. Cuantitativamente, la presentación de dos ponencias deja al descubierto el descuido de la teorización y la filosofía de la educación, ese análisis y reflexión del campo, imperioso para el avance educacional.

En la primera ponencia, Contreras, Baca y Cardona (2018) teorizaron la filosofía utilizada como herramienta metodológica en educación primaria. Se trata de un reclamo de la utilización de este método en un nivel educativo que no siempre se considera; los argumentos son elocuentes y representan a la filosofía más que una actividad discursiva y no solo como contenido de un curso. Además de ser interesantes, también debe encontrar la practicidad, discusión y reflexión, las cuales deben estar presentes en las constantes interrogantes individuales. En relación a la segunda ponencia, sustentada teóricamente con Michel Foucault, Marxen y Greteman anali- 
zaron el poder a través del discurso y cómo es ingerido o introducido en educación a través de las distintas tecnologías, en este caso las farmacéuticas, haciendo un nexo entre la educación y la salud que se empoderan sobre el ser humano. "Ingerir poder" en educación expone una reflexión y análisis de los procesos educativos para percibir si están en la línea de la conservación del statu quo o de la transformación de los individuos y de la sociedad; sin lugar a dudas permite la reflexión filosófica en relación a la salud mental que se vive en los campus universitarios.

Metodológicamente, estas ponencias se apoyan en métodos reflexivos, incentivando el pensamiento dirigido a desarrollar propuestas de trabajo dentro de las aulas para que se conviertan en verdaderos recintos de análisis y para producir ciencia, dejar de ser simples reproductores y operarios por parte de los actores educacionales. Se incide en la teoría de Foucault para profundizar en los espacios escolares, para convertirlos en espacios críticos, de análisis y reflexión, y precisamente a partir de ahí que surjan propuestas para que las relaciones sociales de poder se transformen en relaciones dialógicas y construyan una nueva sociedad.

Fueron siete las contribuciones que se presentaron en el congreso otorgadas al apartado de historia e historiografía de la educación. Una de ellas bajo el nombre "Narrativas: cómo aprendimos a leer y escribir", presentada por Francisco Alberto Pérez, Guillermo Hernández y Jesús Adolfo Trujillo; "La Escuela Secundaria Gustavo L. Talamantes, un proyecto educativo en Ranchería Juárez (1971-1984)", presentada por los mismos autores en diferente orden.

El resto de ponencias de este apartado contaron también con tres autores, dos de los docentes ya mencionados y uno de sus estudiantes (mencionándose aquí solamente el nombre del estudiante, para no ser repetitivo). Una de ellas es "El fundamentalismo religioso mormón en Chihuahua, su devenir histórico y educativo" por Stefany Liddiard; "La peculiaridad del movimiento estudiantil chihuahuense de 1973", de Gerónimo Ontiveros; "El sistema educativo mexicano como base en la construcción del nuevo estado/nación. Elementos pedagógicos y jurídicos. Redes políticas e intelectuales en el Estado de Chihuahua (1857-1910)" de Virginia Verónica Villegas; "La educación media superior en Chihuahua 1827-1973. Una aproximación a los antecedentes para la fundación del sistema Cobach", de Marilyn Georgia Salcido; "La propaganda socialista en Chihuahua durante el mandato de Rodrigo M. Quevedo: un análisis a partir del informe del último año de gobierno", de la autoría de Izabela Tkocz. Como ya se especificó, todas ellas en coautoría con dos de sus docentes.

Llama la atención que las siete ponencias presentadas son de un grupo compacto de la Facultad de Filosofía y Letras de la Universidad Autónoma de Chihuahua. Salvo los ponentes Guillermo Hernández, Francisco Alberto Pérez y Jesús Adolfo Trujillo, el resto de participantes son estudiantes del Doctorado en Educación Artes y Humanidades, siendo los primeros profesores del mismo programa. Con esto se divisa un equipo de profesores y estudiantes que constantemente abonan en su campo, y que en este espacio académico dan a conocer los avances de los trabajos investigativos. El agrupamiento de tres personas por ponencia se debe a la pertenencia de un cuerpo académico consolidado, integrado por los tres profesores mencionados, y en el caso del trabajo con los estudiantes, el orden es que quien elabora la ponencia 154 es el primer autor, seguido de su director de tesis y al final se complementa con otro 
profesor del cuerpo académico, esto por seguir la política académica establecida desde la Subsecretaría de Educación Superior.

Los trabajos de investigación están enmarcarlos en microhistorias del estado de Chihuahua, ya que hacen alusión al rescate de la historia regional. Se trata de investigaciones elaboradas con fuentes primarias o de archivo, acompañadas de entrevistas.

En uno de los casos se dio la continuidad del congreso nacional al internacional con el estudio de la comunidad Le Barón, lo que permite expresar que forman parte de una investigación más amplia y se dieron a conocer productos parciales en cada una. Ambas ponencias de Liddiard, Pérez y Hernández (2018), desde la historiografía, contribuyen a la interpretación de la identidad chihuahuense. Se subraya una línea el entramado de la historia del tiempo pasado con la del tiempo presente, junto a un acercamiento entre los elementos de la historia cultural, justificados desde la psicología social y la sociológica; es decir, con sustento interdisciplinario.

En general, examinando las ponencias desde los paradigmas historiográficos, se observa presentes elementos del historicismo rankeano, con la investigación focalizada en las fuentes primarias de archivo. Existentes, pero en menor cantidad, hay ponencias que evidencian posturas desde la escuela francesa de annales en su tercera etapa, con interpretaciones no solo de hechos, sino de las mentalidades, mitos, ideas e imaginarios sociales presentes en la cultura. Se confirma la apertura y migración del método histórico lógico hacia la metodología interdisciplinaria para trabajar en este campo.

El método histórico lógico, que se observa en los autores (Pérez, Hernández y Trujillo; Trujillo, Hernández y Pérez; Liddiard, Pérez y Hernández; Ontiveros, Pérez y Hernández; Villegas, Hernández y Pérez; Salcido, Trujillo y Hernández; Tkocz Trujillo y Hernández, 2018) permite acercarse a las evidencias, concatenar dichas fuentes y con ello reconstruir el acontecimiento; esto es, se busca dar cuenta del hecho junto a las interpretaciones y valoraciones. Asimismo, la forma de representar la objetividad de las evidencias empíricas se suma a las subjetividades del investigador, quien elabora juicios y valoraciones. Sin embargo, este método se ajusta casi en exclusiva para la historia del tiempo pasado. En la actualidad, este método permanece, pero a la vez se incorporan, con mayor credibilidad, los métodos orales, en donde las fuentes son los propios sujetos, quienes aportan la información de lo investigado, interesando las condiciones de cómo estos sujetos han vivido dicho acontecimiento.

Es una historia cercana a la sociología, ya que tienen cabida en los estudios históricos las interacciones entre sujetos, cercana también a la psicología, para entender esa historia de las mentalidades, de los sujetos actuales, o bien los que se pueden llegar a conocer a través de los documentos. Lo que interesa de esta disciplina es conocer el sentido que las personas le asignan a sus acciones, mitos, imaginarios, deseos y aspiraciones; también la cercanía con la antropología y filosofía buscando el conocimiento ontológico del hombre como homo faber. En fin, lo que se aprecia en las ponencias presentadas en este congreso internacional, y analizadas en este trabajo, es un acercamiento lento, pero novedoso, a la historia, que va de la mano con otras disciplinas, migrando hacia la interdisciplinariedad.

El último apartado de investigación sobre la investigación educativa agrupa ponencias como la llamada "Docentes no heterosexuales: cómo se reconocen en la 
sociedad y qué mecanismos utilizan para sobrevivir en el magisterio. Estado del arte", por Liliana Elizabeth Martínez, del Centro Regional de Educación Integral Benito Juárez, Chihuahua; y una segunda titulada "La formación de subjetividades en una escuela alternativa", presentada por Laura Verónica Herrera y Gerardo Roacho, de la Universidad Pedagógica Nacional del Estado de Chihuahua.

La primera de ellas refiere la poca investigación y casi nula en los campos educativos relacionados con las comunidades lésbico, gay, bisexual, transexual, transgénero, travesti y queer (LGBTTIQ); sin embargo, Martínez (2018) mencionó que aun con el poco estado del conocimiento, se percibe la discriminación y el rechazo de que son víctimas, precisamente por no ser aceptados en sociedades occidentales. El segundo trabajo, Herrera y Roacho (2018) expresaron reflexiones del cómo la tan mencionada reforma educativa -instrumentada en el sexenio pasado y en proceso de derogación a partir del primero de diciembre del 2018- plantea la construcción de subjetividades sujetas por políticas que van en contra de los profesores; en contraposición, se describe que desde los espacios áulicos un grupo de profesores se rebeló e inició la crítica y reflexión para cambiar el estado de lo existente, lo ocurrido en los enfrentamientos y atropellos de los que han sido objeto los profesores.

Ambas ponencias están elaboradas a partir de un proceso documental, reforzadas con entrevistas a profundidad y experiencias con grupos focales, distinguiendo en ellas enfoques cualitativos que consideran la oralidad de los involucrados. Se indica como pendiente, y a manera de colofón, el constante reconocimiento de los espacios educativos como un conglomerado donde conviven personas con diversidad cultural y sexual, a lo que investigadores les han prestado poca atención. Esto se puede afirmar precisamente por los tabúes sociales a nivel micro o macroeducacional.

Los autores de las ponencias se pueden ubicar en la línea del pensamiento pedagógico crítico e innovador, dos categorías que no deben de perder su valor teórico y práctico en el avance de una educación más horizontal e inclusiva. La conclusión es simple y demuestra la urgencia de abordar estas temáticas para no dejarlas invisibles u ocultas; en relación a las subjetividades críticas, se deberá continuar investigando sobre esos procesos dialógicos y críticos.

\section{Clonculsolotes}

La Rediech surgió como resultado de las propuestas investigativas de un grupo magisterial en educación superior. Al inicio centrado en acciones para elaborar los estados del conocimiento de la investigación educativa en el estado de Chihuahua; posteriormente, y ante la necesidad de contar con un espacio académico que trascendiera a lo interinstitucional, que permitiera debates y se diera a conocer a lo macro la producción en materia investigativa, decidieron organizar, a la fecha, cuatro congresos, que han permitido un cúmulo de experiencias en pro del desarrollo educativo de Chihuahua, así como el tránsito e integración de las funciones docentes hacia la investigación.

La mecánica de trabajo instrumentada en los dos congresos analizados es innovadora en cuanto a la presentación de las ponencias. Pasó de ser una presentación 
individual, apoyada por algún material visual o la lectura a la misma ponencia, a una sesión panel en la que cada participante, agrupado por afinidad temática, interviene dialogando y discutiendo sobre las preguntas que el moderador o el público le realizan. Esto ha permitido cambiar de la pasividad anteriormente experimentada a una participación activa tanto de los ponentes como del público asistente.

De manera general, las ponencias fueron presentadas por tres autores, como respuesta a las políticas educativas a nivel superior para el fortalecimiento de los cuerpos académicos, a la obtención de los perfiles Prodep, reconocimientos del Sistema Nacional de Investigadores, estímulos institucionales, entre otros, siendo el trabajo colegiado una forma adecuada para esos fines.

El número de ponencias en el apartado de teoría y filosofía de la educación disminuyeron de tres en el primer congreso a dos en el segundo. Los autores no mostraron continuidad en su línea de investigación, divisándose nuevos actores que abonan al área de conocimiento.

Concentrando el mayor número de ponencias en ambos congresos, los trabajos de la historia de la educación concentraron siete ponencias. De estas, en el primer congreso cinco ponencias fueron presentadas por profesores de la Universidad Autónoma de Chihuahua y una estudiante doctoral; las dos restantes presentadas por profesores del Centro de Investigación y Docencia y del Centro Chihuahuense de Estudios de Posgrado, respectivamente. Durante el segundo congreso, correspondientes a los mismos profesores con más de sus estudiantes doctorales, todos adscritos a la Facultad de Filosofía y Letras, omitidos profesores de otras instituciones. Con ello se puede afirmar que un núcleo de fortalecimiento en esta línea de investigación la consolidan los estudiantes y profesores de dicha universidad, quienes juegan con las reglas de la producción científica de manera colegiada al presentar sus contribuciones por tres autores.

En cuanto a la línea de investigación sobre la investigación educativa se presentaron cuatro ponencias en el primer congreso y dos en el segundo, todas de diferentes ponentes, por lo que se requiere fortalecimiento en grupos de investigación interesados en esta. Asimismo, ambas ponencias del segundo congreso llaman la atención por su temática innovadora: el estudio de la comunidad no heterosexual junto a la poca investigación que existe y la serie de subjetividades como formas alternativas a políticas educativas emanadas del poder, como fue el caso de la reforma educativa del sexenio peñista.

Del análisis realizado de las ponencias de toda el área queda manifiesto la persistencia en la línea de historia de la educación, la continuidad de los profesores y el incremento con los estudiantes del Doctorado en Educación, quienes realizan publicaciones conjuntas y tienen enfoques teóricos que van desde el historicismo rankeano, pasando por la escuela francesa de los annales y la historia interdisciplinaria, lo que promete trabajos críticos que contribuyen al conocimiento de la identidad chihuahuense, basados en el rescate de la historia regional por medio de las microhistorias, con métodos que van desde la investigación en archivos hasta la oralidad, logrando en algunos casos el arte de combinar estas fuentes primarias. 


\section{ReFereErcus}

Bautista, E. (2017). Deserción universitaria en Ingeniería en Sistemas Computacionales. El caso de la Universidad Politécnica Metropolitana de Puebla. RECIE. Revista Electrónica Científica De Investigación Educativa, 3(2), 869-875. Recuperado de http://www.rediech.org/ojs/2017/index.php/recie/article/view/389

Consejo Mexicano de Investigación Educativa. (2015). Memoria electrónica del congreso nacional de investigación educativa. Recuperado de http://www.comie.org.mx/ congreso/memoriaelectronica/v13/seccion4.htm

Contreras, G., Baca, L., y Cardona, A. (2019). La filosofía como método de enseñanza y aprendizaje en la educación primaria. RECIE. Revista Electrónica Científica De Investigación Educativa, 4(2), 867-873. Recuperado de http://www.rediech.org/ ojs/2017/index.php/recie/article/view/404

Chávez, B., Mancera, F., y Rey, L. (2017). La educación física en la ciudad de Chihuahua 1900-1950. RECIE. Revista Electrónica Científica De Investigación Educativa, 3(2), 819-830. Recuperado de http://www.rediech.org/ojs/2017/index.php/recie/article/ view/151

Durán, J. (2016). Derechos Humanos. Abstracción, homogeneización y dominación. RECIE. Revista Electrónica Científica De Investigación Educativa, 3(1), 75-81. Recuperado de http://www.rediech.org/ojs/2017/index.php/recie/article/view/187

Garay, A (2009). Las Areas de Investigación y los Cuerpos Académicos: las tensiones y efectos entre dos espacios de organización de la investigación en la UAM. REencuentro. Análisis de Problemas Universitarios, (55), 18-23.

Hernández, G., Pérez, F., y Trujillo, J. (2016). La escuela unitaria "Agustín de Iturbide" de Sueco, Chihuahua, 1945-1952. IE Revista de Investigación Educativa de la Rediech, 7(12), 7-14. Recuperado de http://www.rediech.org/ojs/2017/index.php/ ie rie rediech/article/view/79

Liddiārd, S., Pérez, F. y Hernández, G. (2017). La diáspora de los mormones fundamentalistas: Colonia Le Barón, Chihuahua. RECIE. Revista Electrónica Científica de Investigación Educativa, 3(2), 839-845. Recuperado de http://www. rediech.org/ojs/2017/index.php/recie/article/view/385

Liddiard, S., Pérez, F., y Hernández, G. (2018). El fundamentalismo religioso mormón en Chihuahua, su devenir histórico y educativo. RECIE. Revista Electrónica Científica de Investigación Educativa, 4(1), 73-84. Recuperado de http://www.rediech.org/ ojs/2017/index.php/recie/article/view/305

Martínez, L. (2018). Docentes no heterosexuales: cómo se reconocen en la sociedad y qué mecanismos utilizan para sobrevivir en el magisterio. Estado del arte. RECIE. Revista Electrónica Científica de Investigación Educativa, 4(1), 85-94. Recuperado de http:// www.rediech.org/ojs/2017/index.php/recie/article/view/352

Marxen, E., y Greteman, A. (2019). Ingerir poder. RECIE. Revista Electrónica Científica De Investigación Educativa, 4(2), 875-886. Recuperado de http://www.rediech.org/ ojs/2017/index.php/recie/article/view/362

Morán, P. (1993). La vinculación docencia investigación como estrategia pedagógica. Perfiles Educativos, (61)

Ontiveros, G., Pérez F., y Hernández, G. (2018). La peculiaridad del movimiento estudiantil chihuahuense de 1973. RECIE. Revista Electrónica Científica De Investigación Educativa, 4(1), 23-34. Recuperado de http://www.rediech.org/ ojs/2017/index.php/recie/article/view/289

Pérez, F., Hernández, G., y Trujillo, J. (2018). Narrativas: Cómo aprendimos a leer y escribir. RECIE. Revista Electrónica Científica De Investigación Educativa, 4(1), 4754. Recuperado de http://www.rediech.org/ojs/2017/index.php/recie/article/view/293 
Pérez, F., Hernández, G., y Trujillo, J. (2016). Al rescate de contenidos históricoeducativos: la hacienda de Coyotillos. RECIE. Revista Electrónica Científica De Investigación Educativa, 3(1), 23-29. Recuperado de http://www.rediech.org/ ojs/2017/index.php/recie/article/view/181

Ponce, F. (2017). Historia Cultural de la Educación Especial en Chihuahua: Transformación de su cultura escolar 1970-2015. RECIE. Revista Electrónica Científica De Investigación Educativa, 3(2), 855-867. Recuperado de http://www. rediech.org/ojs/2017/index.php/recie/article/view/388

Ponce, H. (2016). Evaluación de los Índices de Reprobación de la Universidad usando Intervalos de Confianza. RECIE. Revista Electrónica Científica De Investigación Educativa, 3(1), 47-57. Recuperado de http://www.rediech.org/ojs/2017/index.php/ recie/article/view/184

Programa de Mejoramiento del Profesorado. (2018) Cuerpo Académico, conceptos básicos. Recuperado de https://promep.sep.gob.mx/cal/Conceptos2.html

Ramos, J., Loya, C., y Vázquez, A. (2017). La instrucción pública en la nueva escuela de primeras letras en la ciudad de Chihuahua en 1869. Un intento de innovar. RECIE. Revista Electrónica Científica De Investigación Educativa, 3(2), 831-837. Recuperado de http://www.rediech.org/ojs/2017/index.php/recie/article/view/382

Red de Investigadores Educativos Chihuahua. (2017) Revista de investigación educativa. Recuperado de http://www.rediech.org/ojs/2017/index.php/ie_rie_rediech/index

Red de Investigadores Educativos Chihuahua. (2018). IV Congreso internacional de investigación educativa. Recuperado de: http://www.rediech.org/inicio/index.php/ congreso/itemlist/category/19-eventos? start $=16$

Reyes, B. (2017). ¿Educación moral para la autonomía? Fundamentos y contradicciones. RECIE. Revista Electrónica Científica De Investigación Educativa, 3(2), 847-853. Recuperado de http://www.rediech.org/ojs/2017/index.php/recie/ article/view/387

Roacho, G. y Herrera, L. (2018). Formación de subjetividades en una escuela alternativa. RECIE. Revista Electrónica Científica De Investigación Educativa, 4(1), 599-608. Recuperado de http://www.rediech.org/ojs/2017/index.php/recie/article/ view/406

Rodríguez, N. (2016) Ponencia presentada en el III Congreso Nacional de Investigación Educativa organizado por a Rediech.

Rodríguez, N. (2016). La formación del ciudadano desde la hermenéutica de lo ausente. RECIE. Revista Electrónica Científica De Investigación Educativa, 3(1), 59-64. Recuperado de http://www.rediech.org/ojs/2017/index.php/recie/article/view/185

Salcido, M., Trujillo, J., y Hernández, G. (2018). La Educación Media Superior en Chihuahua 1827-1973. Una aproximación a los antecedentes para la fundación del sistema COBACH. RECIE. Revista Electrónica Científica De Investigación Educativa, 4(1), 35-46. Recuperado de http://www.rediech.org/ojs/2017/index.php/ recie/article/view/292

Tkocz, I., Trujillo, J., y Hernández, G. (2018). La propaganda socialista en Chihuahua durante el mandato de Rodrigo M. Quevedo: un análisis a partir del informe del último año de gobierno. RECIE. Revista Electrónica Científica De Investigación Educativa, 4(1), 55-72. Recuperado de http://www.rediech.org/ojs/2017/index.php/ recie/article/view/299

Torres, J. (2016). Educación vacía: un reto en la zona del bajío. RECIE. Revista Electrónica Científica De Investigación Educativa, 3(1), 31-37. Recuperado de http:// www.rediech.org/ojs/2017/index.php/recie/article/view/182

Trujillo, J., Hernández, G., y Pérez, F. (2019). La Escuela Secundaria Gustavo L. Talamantes, un proyecto educativo en Ranchería Juárez (1971-1984). RECIE. Revista Electrónica Científica De Investigación Educativa, 4(2), 909-920. Recuperado de http://www.rediech.org/ojs/2017/index.php/recie/article/view/453 
Trujillo, J., Pérez, F., y Hernández, G. (2016). Memoria e identidad. La recuperación de la historia educativa de Ranchería Juárez a través de sus protagonistas. RECIE. Revista Electrónica Científica De Investigación Educativa, 3(1), 65-74. Recuperado de http://www.rediech.org/ojs/2017/index.php/recie/article/view/186

Villegas, V., Hernández, G. y Trujillo, J. (2019). El Sistema Educativo Mexicano como base en la construcción del nuevo Estado/Nación. Elementos pedagógicos y jurídicos. Redes políticas e intelectuales en el Estado de Chihuahua (1857-1910). RECIE. Revista Electrónica Científica De Investigación Educativa, 4(2), 887-896. Recuperado de de http://www.rediech.org/ojs/2017/index.php/recie/article/view/343 